\title{
Landauer-Büttiker study of the anomalous Hall effect
}

\author{
Maria Silvia Garelli and John Schliemann \\ Institute for Theoretical Physics, University of Regensburg, D-93040 Regensburg, Germany \\ (Received 1 July 2009; revised manuscript received 19 August 2009; published 20 October 2009)
}

\begin{abstract}
We report on Landauer-Büttiker studies of anomalous Hall transport in a two-dimensional electron gas with Rashba spin-orbit coupling and a magnetization provided by localized magnetic moments. Our system is described by a discretized tight-binding model in a four-terminal geometry. We consider both the case of magnetically disordered systems as well as ballistic transport in disorder-free systems with spatially homogeneous magnetization. In the latter case we investigate both out-of-plane and in-plane magnetizations. We numerically establish a close connection between singularities in the density of states and peaks in the Hall conductance close to the lower band edge. Consistent with previous theoretical studies based on diagrammatic perturbation expansions, these peaks occur at Fermi energies where only the lower dispersion branch is occupied. Moreover, for large magnetization the Hall conductance is, along with the density of states, suppressed. This numerical finding can be understood from analytical properties of the underlying model in the limit of an infinite system.
\end{abstract}

DOI: 10.1103/PhysRevB.80.155321

PACS number(s): 72.15.Eb, 72.20.Dp

\section{INTRODUCTION}

The anomalous Hall effect (AHE) is the subject of a long standing and partially still ongoing theoretical debate. ${ }^{1-7}$ It amounts in a Hall conductivity which is not due to an external magnetic field but the result of the magnetization of a solid. A large portion of the renewed interest in this phenomenon is generated by research on ferromagnetic semiconductors. ${ }^{8,9}$ In general, it is common to distinguish between two types of mechanisms for the AHE, both relying on spin-orbit interaction. The extrinsic mechanism requires the presence of impurities or other imperfections and is based on contributions to spin-orbit coupling from such scattering potentials. This spin dependence of the effective scattering potential gives rise to the skew-scattering ${ }^{2}$ and the side-jump ${ }^{3}$ contributions to the anomalous Hall conductivity. The intrinsic mechanism is independent of scattering centers and is a result of the spin-orbit-coupled electronic band structure, where the spin-orbit interaction stems from the ordered crystal lattice itself.

Among many different systems, the case of a twodimensional semiconductor electron gas with an intrinsic effective spin-orbit coupling of the Rashba type ${ }^{10}$ has attracted considerable theoretical interest ${ }^{11-23}$ and was also studied experimentally in an $n$-doped II-VI semiconductor heterostructure containing manganese ions. ${ }^{24}$ The theoretical investigations have considered both the intrinsic effect ${ }^{17,18}$ as well as combinations of intrinsic and extrinsic mechanisms. ${ }^{11-16,19-23}$ In the present work we shall concentrate on the purely intrinsic AHE.

An important tool for the theoretical description of transport in such mesoscopic systems is given by the LandauerBüttiker formalism. ${ }^{25,26}$ In this paper we report on numerical studies within this approach on Hall transport in a twodimensional electron gas (2DEG) with Rashba spin-orbit interaction and magnetic impurities. Such an investigation of the AHE seems to be missing in the previous literature although several studies of this kind on the related spin Hall effect are available. ${ }^{27-35}$
This paper is organized as follows. In Sec. II we describe important properties of our underlying model and outline the Landauer-Büttiker approach to transport in such systems. More specific information on Green's function in semiinfinite leads used in our study can be found in Appendix. In Sec. III we present our numerical results covering both ballistic transport and transport in magnetically disordered systems. We close with conclusions and an outlook in Sec. IV.

\section{MODEL AND APPROACH}

We consider a two-dimensional gas of noninteracting electrons with spin-orbit interaction of the Rashba type. ${ }^{10}$ Additionally, the electron spin is coupled to magnetic impurities.

\section{A. Continuum model}

The generic single-particle Hamiltonian for the continuum system reads

$$
\mathcal{H}=\frac{\vec{p}^{2}}{2 m^{*}}+\frac{\alpha}{\hbar}\left(p_{x} \sigma^{y}-p_{y} \sigma^{x}\right)+\vec{\Delta} \cdot \vec{\sigma} .
$$

Here $\vec{p}$ is the electron momentum, $m^{*}$ its effective band mass, and the Pauli matrices $\vec{\sigma}$ describe the electron spin. The strength of the spin-orbit interaction is described by the Rashba parameter $\alpha$ and $\vec{\Delta}$ is the effective Zeeman splitting due to the coupling of the electron spin to magnetic impurities. In general, this quantity will be position dependent, $\vec{\Delta}$ $=\vec{\Delta}(\vec{r})$. However, it is also instructive to consider the case of spatially constant magnetization corresponding to an uniform impurity polarization. In the following we will consider both the case of a homogeneous magnetization perpendicular to the plane of the electron gas, as well systems with in-plane magnetization. We note that an in-plane magnetization can also be interpreted as a genuine magnetic field which couples in a strictly two-dimensional situation only to the spin of the electron but not to its orbital degrees of freedom. For a ho- 
mogeneous magnetization the eigenstates are given by plane waves and the energy of a given wave vector $\vec{k}=\vec{p} / \hbar$ reads

$$
\varepsilon(\vec{k})=\frac{\hbar^{2} k^{2}}{2 m^{*}} \pm \sqrt{\left(-\alpha k_{y}+\Delta_{x}\right)^{2}+\left(\alpha k_{x}+\Delta_{y}\right)^{2}+\Delta_{z}^{2}} .
$$

Let us first discuss the case of a purely perpendicular magnetization, $\vec{\Delta}=(0,0, \Delta)$. Here one finds that, provided that the energy scale of the Rashba coupling $\varepsilon_{R}:=m^{*} \alpha^{2} / \hbar^{2}$ is larger than the Zeeman coupling,

$$
\varepsilon_{R}>|\Delta|,
$$

the lower dispersion branch in Eq. (2) has a minimum at finite $k=k_{\text {min }}$

$$
k_{\text {min }}=\frac{1}{|\alpha|} \sqrt{\varepsilon_{R}^{2}-\Delta^{2}}
$$

with minimum energy

$$
\varepsilon_{\text {min }}=\varepsilon_{-}\left(k_{\text {min }}\right)=-\frac{1}{2} \varepsilon_{R}-\frac{1}{2} \frac{\Delta^{2}}{\varepsilon_{R}} .
$$

This dispersion minimum at finite wave vector leads to a van Hove singularity in the electronic density of states (DOS) at the bottom of the lower branch, $\varepsilon \rightarrow \varepsilon_{\text {min }}+0$. Explicitly, the density of states is given by

$$
D(\varepsilon)=\left\{\begin{array}{cc}
0 & \varepsilon<\varepsilon_{\text {min }} \\
\frac{m^{*}}{\pi \hbar^{2}} \sqrt{\frac{\varepsilon_{R}}{2\left(\varepsilon-\varepsilon_{\text {min }}\right)}} & \varepsilon_{\text {min }} \leq \varepsilon<-|\Delta| \\
\frac{m^{*}}{2 \pi \hbar^{2}}\left(1+\sqrt{\frac{\varepsilon_{R}}{2\left(\varepsilon-\varepsilon_{\text {min }}\right)}}\right) & -|\Delta| \leq \varepsilon<|\Delta| \\
\frac{m^{*}}{\pi \hbar^{2}} & |\Delta| \leq \varepsilon
\end{array}\right.
$$

This quantity has obviously a square-root singularity at $\varepsilon \rightarrow \varepsilon_{\text {min }}+0$. As we shall see below, such singularities are intimately linked to the observation of anomalous Hall transport. Note also that $D(\varepsilon)$ is discontinuous (but finite) at $\varepsilon= \pm|\Delta|$. These discontinuities vanish for $\Delta=0$. On the other hand, if the Zeeman coupling dominates over the spin-orbit interaction,

$$
\varepsilon_{R}<|\Delta|,
$$

the energy dispersion branches have stationary points only at zero wave vector. Here no van Hove singularity occurs in the density of states, apart from a steplike behavior at minimum energy. These two cases $\varepsilon_{R} \gtrless|| \Delta \mid$ are illustrated in the left panel of Fig. 1. Note that for $\varepsilon_{R}>|\Delta|$ the avoided crossing at $k=0$ does not lead to a van Hove singularity since these stationary points occur at vanishing wave vector.

In the case of an arbitrary magnetization direction, $\vec{\Delta}$ $=\left(\Delta_{x}, \Delta_{y}, \Delta_{z}\right)$, a closer analysis shows that the stationary points of the dispersion (fulfilling $\partial \varepsilon_{ \pm} / \partial \vec{k}=0$ ) lie at wave vectors perpendicular to the in-plane projection of the magnetization,

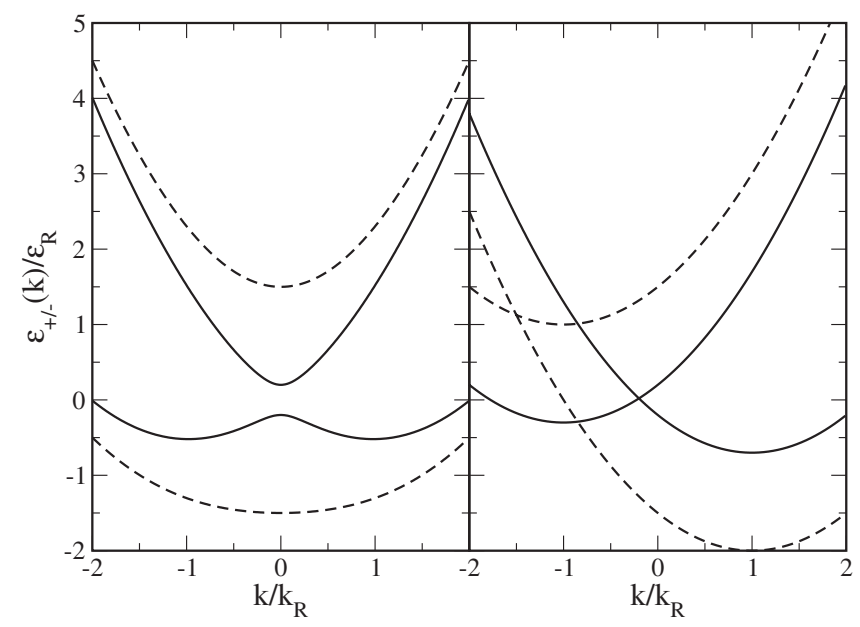

FIG. 1. Energy dispersion $\varepsilon_{+}(\vec{k})$ for $\Delta=0.2 \varepsilon_{R}$ (solid lines) and $\Delta=1.5 \varepsilon_{R}$ (interrupted lines). Left panel: perpendicular magnetization $\vec{\Delta}=(0,0, \Delta)$. Right panel: in-plane magnetization $\vec{\Delta}$ $=\left(\Delta_{x}, \Delta_{y}, 0\right)$ with the wave vector $\vec{k}$ being orthogonal to $\vec{\Delta}, \vec{\Delta} \cdot \vec{k}$ $=0$. The energies are given in units of the Rashba energy $\varepsilon_{R}$ while the wave vector is measured in units of the inverse "Rashba wave length" $k_{R}=m^{*} \alpha / \hbar^{2}$.

$$
\vec{k} \cdot \vec{\Delta}=0
$$

However, since this very general case does not seem to allow for further explicit results, we shall concentrate on a purely in-plane magnetization, $\vec{\Delta}=\left(\Delta_{x}, \Delta_{y}, 0\right)$. Here the stationary points of the dispersion correspond to energy minima and lie at wave vectors

$$
\vec{k}_{\text {min }}^{ \pm}= \pm \frac{m^{*} \alpha}{\hbar^{2}} \frac{\left(-\Delta_{y}, \Delta_{x}\right)}{\Delta} .
$$

The absolute minimum is given by

$$
\varepsilon_{\text {min }}^{-}=\varepsilon_{-}\left(\vec{k}_{\text {min }}^{-}\right)=-\frac{1}{2} \varepsilon_{R}-\Delta
$$

(assuming $\alpha>0$ ) while another minimum occurs at $\vec{k}=\vec{k}_{\text {min }}^{+}$ with

$$
\varepsilon_{\text {min }}^{+}=\varepsilon_{ \pm}\left(\vec{k}_{\text {min }}^{+}\right)=-\frac{1}{2} \varepsilon_{R}+\Delta
$$

where the plus (minus) sign in $\varepsilon_{ \pm}(\cdot)$ applies if $\Delta>\varepsilon_{R}$ $\left(\Delta<\varepsilon_{R}\right)$. These two cases are depicted in the right panel of Fig. 1. The dispersion minima at finite wave vector $\vec{k}=\vec{k}_{\text {min }}^{ \pm}$ are physically easily understood: In the case $\varepsilon=\varepsilon_{\text {min }}^{-}$, the Zeeman field the and spin coupling provided by the Rashba interaction are parallel to each other leading to an energetic minimum for the appropriate spin direction while for the higher minimum $\varepsilon=\varepsilon_{\text {min }}^{+}$these couplings are antiparallel. Note also that these energetic minima remain at finite wave vector for arbitrarily large magnetization. Therefore, differently from the case of perpendicular magnetization, the van Hove singularities in the density of states do not vanish for large Zeeman coupling. 


\section{B. Discrete system}

The standard discretized version of Hamiltonian (1) on a square lattice reads

$$
\begin{aligned}
\mathcal{H}= & -t \sum_{m, n ; \alpha}\left[c_{m, n ; \alpha}^{\dagger} c_{m+1, n ; \alpha}+c_{m, n ; \alpha}^{\dagger} c_{m, n+1 ; \alpha}+\text { H.c. }\right] \\
& +\lambda \sum_{m, n ; \alpha, \beta}\left[-i c_{m, n ; \alpha}^{\dagger} \sigma_{\alpha, \beta}^{x} c_{m, n+1 ; \beta}+i c_{m, n ; \alpha}^{\dagger} \sigma_{\alpha, \beta}^{y} c_{m+1, n ; \beta}\right. \\
& + \text { H.c }]+\sum_{m, n ; \alpha, \beta} c_{m, n ; \alpha}^{\dagger} \vec{\Delta}_{m, n} \cdot \vec{\sigma}_{\alpha, \beta} c_{m, n ; \beta} .
\end{aligned}
$$

Here $m$ and $n$ label lattice locations with respect to the $x$ and $y$ axis, respectively, and $\alpha$ and $\beta$ are spin indices. The hopping parameter $t$ is related to the effective mass $m^{*}$ and the lattice constant $a$ via $t=\hbar^{2} / 2 m^{*} a^{2}$, and the parameter $\lambda$ is given by $\lambda=\alpha / 2 a$. To give a specific example, for a host material such as gallium arsenide we have an effective band mass of $m^{*}=0.067 m_{0}$ (with $m_{0}$ being the free-electron mass) and a lattice spacing of $a=5.6 \AA$ leading to a hopping parameter $t=1.8 \mathrm{eV}$. Typical values for the Rashba parameter are of order $0.1 \mathrm{eV} \AA$ such that we have typically $\lambda$ $\approx 0.01 t$.

For a spatially homogeneous impurity polarization of arbitrary direction, $\vec{\Delta}_{m, n}=\left(\Delta x, \Delta y, \Delta_{z}\right)$ the energy dispersions are given by

$$
\begin{aligned}
\varepsilon_{ \pm}(\vec{k})= & -2 t\left[\cos \left(k_{x} a\right)+\cos \left(k_{y} a\right)\right] \pm\left\{\left[\Delta_{x}-2 \lambda \sin \left(k_{y} a\right)\right]^{2}\right. \\
& \left.+\left[\Delta_{y}+2 \lambda \sin \left(k_{x} a\right)\right]^{2}+\Delta_{z}^{2}\right\}^{1 / 2}
\end{aligned}
$$

In order to make contact to the continuum model, one has to evaluate this dispersion for small wave vector, $k a \ll 1$, corresponding to the lower band edge, where it reproduces Eq. (2) up to a rigid shift of $(-4 t)$ which is just half of the bandwidth in the absence of magnetization.

However, these dispersion relations, Eq. (13), of the discrete system lead to very intricate conditions for stationary points which do not seem to be explicitly solvable. We therefore concentrate on the case of a homogeneous polarization perpendicular to the plane, $\vec{\Delta}_{m, n}=(0,0, \Delta)$. Here the lower branch leads again to a singularity in the density of states provided that

$$
2 \lambda^{2}>t|\Delta|
$$

which is exactly the same as the condition (3).

\section{Hall bridge and Landauer-Büttiker formalism}

The above discretized system described by Hamiltonian (12) is studied as the central region of a four-terminal Hall bridge shown in Fig. 2. In the following, we will investigate this system consisting of its central part and ideal semiinfinite leads without spin-orbit coupling and magnetization using the Landauer-Büttiker formalism. ${ }^{25,26}$ We now briefly summarize the most important features of this method as applied to the calculation of the Hall conductivity.

Within the Landauer-Büttiker approach, the Hall conductivity is given by

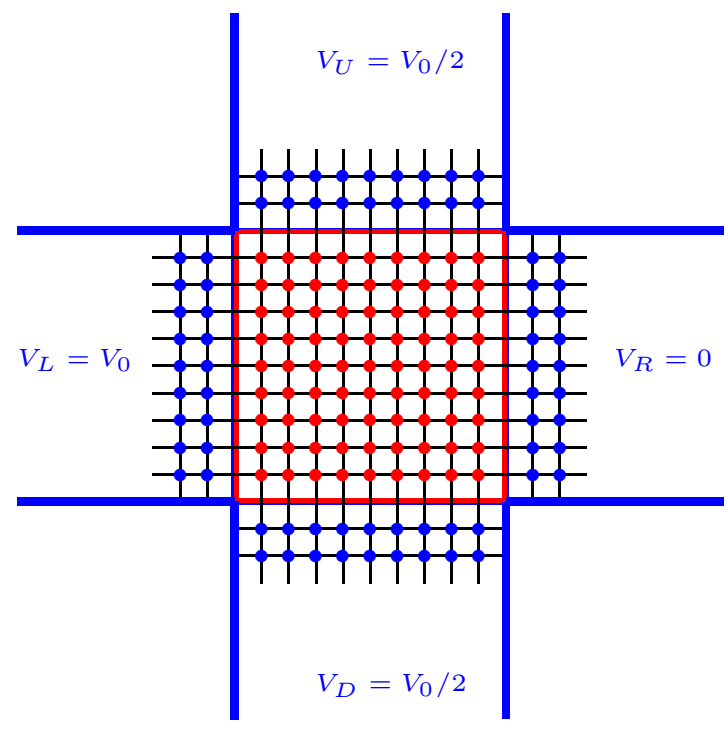

FIG. 2. (Color online) Four-terminal Hall bridge. The central region is described by the discretized Hamiltonian (12) incorporating Rashba spin-orbit coupling and magnetic impurities.

$$
\sigma_{H}=\frac{I_{U}}{V_{L}-V_{R}}=-\frac{1}{2} G_{U L}+\frac{1}{2} G_{U R},
$$

where $I_{U}$ is the current flowing into lead up, and $G_{U L}$ and $G_{U R}$ are the conductances between lead up and left and between lead up and right, respectively. These quantities can be calculated via the following equation:

$$
I_{p}=\sum_{q} G_{p q}\left(V_{p}-V_{q}\right)
$$

which describes the current flowing in lead $p$, where

$$
G_{p q}=\frac{e^{2}}{h} T_{p q}\left(\varepsilon_{f}\right)
$$

is the charge conductance between leads $p$ and $q$. This quantity is proportional to the transmission function $T_{p q}$ defined as

$$
T_{p q}=\operatorname{tr}\left[\Gamma_{p} G^{r} \Gamma_{q} G^{a}\right],
$$

where the retarded (advanced) Green's function $G^{r(a)}$ and the linewidth function $\Gamma$ enter. These functions are the heart of the Landauer-Büttiker approach. Indeed, both Greens's functions

$$
G^{r}=\left[G^{a}\right]^{\dagger}=\left(\varepsilon_{f}-H-\sum_{q} \Sigma_{q}^{r}\right)^{-1}
$$

and

$$
\Gamma_{q}=i\left[\Sigma_{q}^{r}-\Sigma_{q}^{a}\right]
$$

depend on the retarded (advanced) self-energy $\Sigma^{r(a)}$. The retarded $\Sigma_{q, \mu}^{r}$ and the advanced $\Sigma_{q, \mu}^{a}=\Sigma_{q, \mu}^{r^{\dagger}}$ self-energy terms describe the coupling between the $2 \mathrm{DEG}$ in the central region and the four ideal semi-infinite leads and can be formulated as 


$$
\Sigma_{q, \mu}^{r}(i, j)=t^{2} g_{q, \mu}^{r}\left(p_{i}, p_{j}\right),
$$

where $g_{q, \mu}^{r}$ is the Green's function of the isolated semiinfinite lead. The only terms which enter into the self-energy are the coupling terms between each lead and the 2DEG central region. The coupling between a lead and the central system give rise to a coupling matrix which is nonzero only for adjacent points $i$ (lying at the edge of the 2DEG) and $p_{i}$ (lying at the lead's edge which faces the 2DEG). Since the lead Green's function can be evaluated analytically, ${ }^{25,26}$ the self-energy method can be exploited to deal with an infinite system, such as a semi-infinite lead, by calculating only the Green's function of a finite region. The analytical expression of our Green's function is derived in Appendix.

Another important quantity which can be calculated within the Landauer-Büttiker formalism is the DOS given by

$$
D(\varepsilon)=\frac{1}{2 \pi N^{2} a^{2}} \operatorname{tr}[A(\varepsilon)]=-\frac{1}{2 \pi N^{2} a^{2}} \operatorname{tr}\left[\operatorname{Im}\left(G^{r}\right)\right],
$$

where $A(\varepsilon)=i\left[G^{r}-G^{a}\right]$ is the spectral function and $N^{2}$ is the number of lattice sites in the central region taken to be a square. The above expression is identical to another standard textbook result,

$$
\begin{gathered}
D(\varepsilon)=\frac{1}{(2 \pi)^{2}} \int d^{2} \vec{k} \delta[\varepsilon-\varepsilon(\vec{k})] \\
=\frac{1}{(2 \pi)^{2}} \oint_{\varepsilon(\vec{k})=\varepsilon} d k \frac{1}{\left|\nabla_{\vec{k}} \varepsilon\right|},
\end{gathered}
$$

which can be used to derive, e.g., Eq. (6). For an infinite 2DEG with dispersion $\varepsilon(\vec{k})=-2 t\left[\cos \left(k_{x} a\right)+\cos \left(k_{y} a\right)\right]$ the DOS can be calculated analytically leading to a logarithmic divergence at $\varepsilon=0$ and a saturation to constant values at the edges of the band, i.e., $D(\varepsilon= \pm 4 t)=\frac{1}{4 \pi} t a^{2}$. On the other hand, in the case of a finite 2 DEG central region and no coupling to the leads, the DOS is just given by a sum of $\delta$-peaks for values of $\varepsilon_{f}$ which match the eigenvalues of the Hamiltonian of the central conductor. As we shall see below, the anomalous Hall conductance is closely related to the DOS.

\section{RESULTS}

Let us now describe our numerical results based on the Landauer-Büttiker formalism outlined before. We first concentrate on disorder-free ballistic systems.

\section{A. Ballistic Hall transport}

Here we present our results for a disorder-free central region with homogeneous magnetization $\vec{\Delta}$. We will both cover the case of magnetization perpendicular to the plane of the $2 \mathrm{DEG}, \vec{\Delta}=(0,0, \Delta)$ and the case of in-plane magnetization of various directions. In the latter scenario, the magnetization $\vec{\Delta}$ can also be interpreted as a proper magnetic field $\vec{B}$ which, in a strictly two-dimensional system, couples only

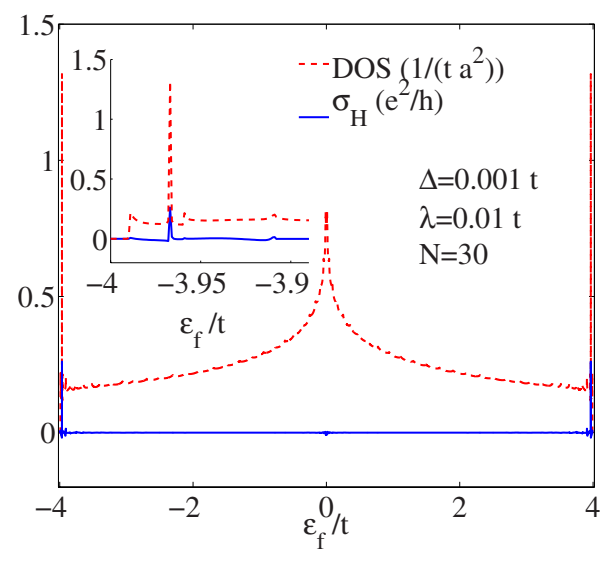

FIG. 3. (Color online) Hall conductance and DOS for a Zeeman coupling $\Delta=0.001 t$ and a Rashba parameter $\lambda=0.01 t$ as a function for Fermi energy $\varepsilon_{f} \in[-4 t-\Delta, 4 t+\Delta]$ and a linear system size of $N=30$. The inset shows the behavior of the Hall conductance and the DOS close to the lower band edge. Both quantities are characterized by a simultaneous peak.

to the spin but not to the orbital degrees of freedom of charge carriers.

\section{Magnetization perpendicular to the $2 D E G$}

Let us first turn to the case of systems magnetized perpendicularly to the plane of the 2DEG. We consider a magnetization $\vec{\Delta}=(0,0, \Delta)$ and have evaluated the Hall conductance and the density of states according to Eqs. (15) and (22), respectively. As is must be, the Hall conductance vanishes for zero magnetization since the conductances $G_{U L}$ and $G_{U R}$ are identical and cancel out. With a finite Zeeman coupling, however, a charge current $I_{U}$ starts to flow in lead up signaling a finite Hall conductance. Figure 3 shows the Hall conductance along with the DOS for a Zeeman coupling $\Delta$ $=0.001 t$ and a Rashba parameter of $\lambda=0.01 t$ as a function of Fermi energy $\varepsilon_{f} \in[-4 t-\Delta, 4 t+\Delta]$. Both quantities plotted are perfectly symmetric with respect to the band center $\varepsilon_{f}$ $=0$. The Hall conductance is characterized by an oscillatory behavior over the entire energy range with particularly dominating peaks near the band edges; a smaller peak occurs also at the band center. On the other hand, the DOS shows the predicted logarithmically divergence at $\varepsilon_{f}=0$ and in addition even more pronounced singularities near the band edges at exactly the same positions as the peaks of the Hall conductance.

As we wish to make contact to previous theoretical investigations on anomalous Hall transport in 2DEGs described by continuum models, ${ }^{11-23}$ we will concentrate in the following on the peaks of Hall conductance and DOS close to the lower band edge. In Fig. 4 we have plotted both quantities near the lower band edge for the same Rashba parameter and system size as before but different Zeeman couplings $\Delta$. As seen in the figure, extrema of the DOS and the Hall conductance occur at the same position in energy, independently of the regime of Zeeman coupling. The close correspondence between extrema of the DOS and the Hall conductance will be an important finding for our further analysis of anomalous 


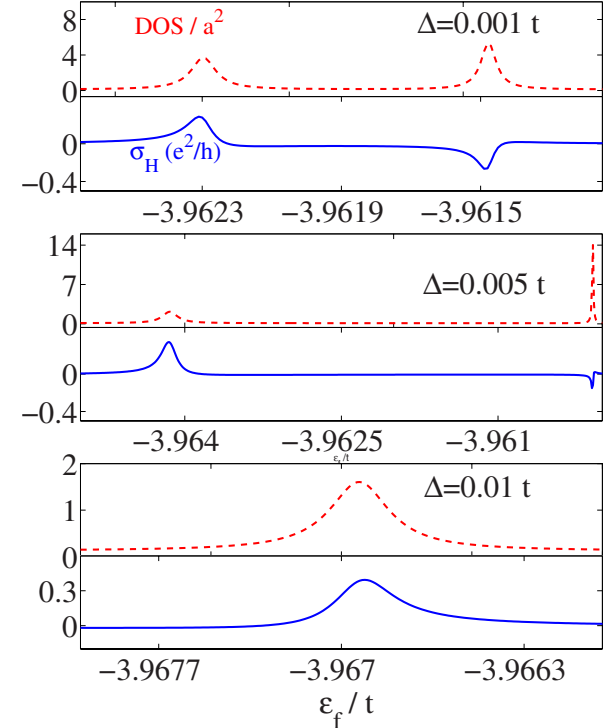

FIG. 4. (Color online) DOS (red dashed line) in units $1 / t a^{2}$ and Hall conductance (blue solid line) in units of $e^{2} / h$ near the lower band edge for the same system size and Rashba coupling as in Fig. 3 but various Zeeman couplings $\Delta$. In all cases an obvious correspondence between the extrema DOS and Hall conductance occurs.

Hall transport. Note that the maxima of the DOS at lower energies become weaker with increasing Zeeman coupling $\Delta$. Such a behavior can indeed be expected from the analytically accessible properties of the infinite system discussed in Sec. II. Here the divergent van Hove singularities in the DOS vanish if the magnetization dominates the spin-orbit coupling. The systems investigated in this work numerically are obviously different as they are finite and coupled to semiinfinite leads lacking spin-orbit interaction. However, the above observations shall still guide our intuition regarding the interplay between magnetization and spin-orbit coupling.

Figure 5 displays the Hall conductance near the bottom of the band for a Rashba parameter of $\lambda=0.01 t$ and various Zeeman couplings $\Delta$ with the linear system size varying from $N=30$ to 50 . For small magnetization $\Delta \lesssim 0.001 t$ the height of the Hall conductance peaks is approximately independent of the system size while for larger Zeeman couplings $0.001 \leqq \Delta \leqq 0.0075 t$ slightly grows with increasing system size. For even larger $\Delta \gtrsim 0.0075 t$ a decrease is observed for large system sizes. This qualitative behavior persists in a range of Rashba parameters $\lambda \in[0.005 t, 0.02 t]$ with the above threshold values for the Zeeman coupling $\Delta$ being approximately unchanged. While our above finite-size data for the height of the Hall conductance peaks does not seem to allow for an unambiguous extrapolation to the thermodynamic limit, the suppression of the Hall conductance at large Zeeman splittings $\Delta \gtrsim 0.0075 t$ is certainly consistent with the analytical observations in the infinite system. At large Zeeman couplings, the singularity in the DOS close to the band edge disappears and, in turn, the Hall conductance vanishes.

Moreover, as also seen in Fig. 5, for all Zeeman couplings, the position $\varepsilon_{N}^{*}$ of the peak shifts to lower energies, i.e., toward the bottom of the band, with increasing system size. Figure 6 shows the finite-size behavior of the peak po-

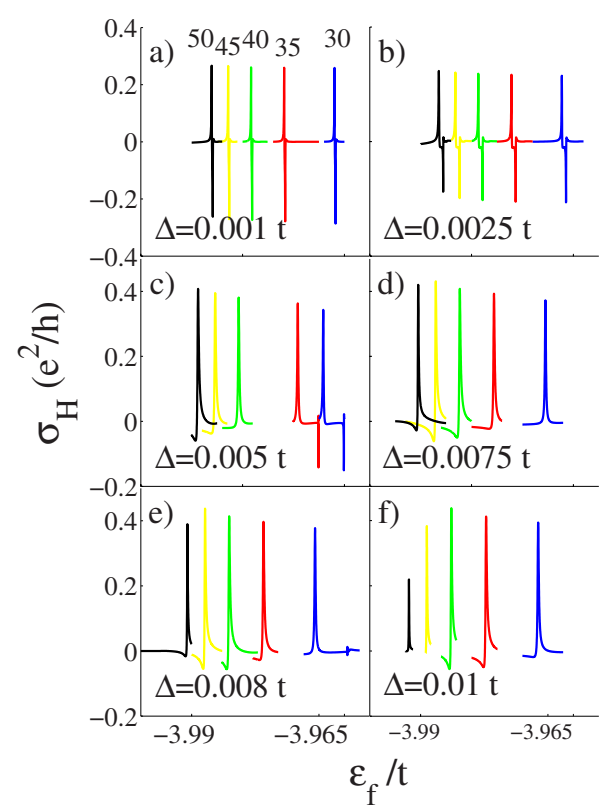

FIG. 5. (Color online) Hall Conductance near the bottom of the band for a Rashba parameter of $\lambda=0.01 t$ and various Zeeman couplings $\Delta$. The linear system size varies from $N=30$ (blue, right), $N=35$ (red), $N=40$ (green), $N=45$ (yellow), and $N=50$ (black, left).

sition for same six data sets as in Fig. 5. All data sets can be smoothly fitted by an exponential function which allows for an extrapolation to the limit of an infinite system, $\varepsilon^{*}=\lim _{N \rightarrow \infty} \varepsilon_{N}^{*}$. The dependence of $\varepsilon^{*}$ on the magnetization $\Delta$ is shown in Fig. 7. Clearly, $\varepsilon^{*}$ linearly decreases with increasing $\Delta$. Note, however, that for large Zeeman splitting the Hall conductance peaks are suppressed with increasing system size although their position can still be followed as a function of $N$. On the other hand, even for the smallest Zeeman gap of $\Delta=0.001 t$ considered here, the infinite-volume peak position $\varepsilon^{*}$ lies at an energy where only the lower dispersion branch of Eq. (13) is occupied. This is in accordance with recent theoretical predictions based on diagrammatic perturbation theory by Nunner et al. ${ }^{18,20}$ who concluded that a finite Hall conductance can only occur at low Fermi energies such that only the lower subband is occupied.

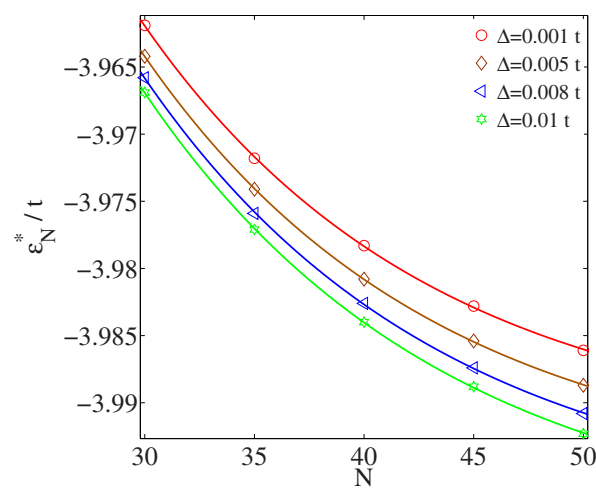

FIG. 6. (Color online) Dependence of the position $\varepsilon_{N}^{*}$ of the Hall conductance peak on the system size for same data sets as in Fig. 5. The Rashba parameter is $\lambda=0.01 t$. 


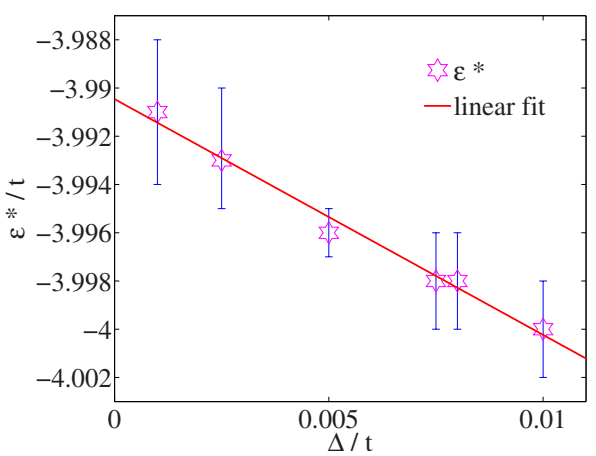

FIG. 7. (Color online) Energy position $\varepsilon^{*}$ of the Hall conductance peak at the lower band edge extrapolated to an infinite system versus the magnetic coupling $\Delta$ : the dependence is well fitted by a linear function.

\section{In-plane magnetization}

We now turn to the case of magnetic impurities polarized in the plane of the 2DEG. As shown in Fig. 2, the Hall current is measured along the $y$ direction in leads up and down while the Hall voltage is applied along the $x$ direction between leads left and right. Here we do not observe a Hall current if the in-plane magnetization is parallel to the voltage since here for charge carriers with wave vector along the $y$ direction the spin coupling resulting from the Rashba interaction and the Zeeman coupling are just parallel to each other. However, a finite Hall current can occur for other inplane directions of the magnetization.

Figure 8 shows the Hall conductance for several directions of magnetization. For magnetization along the $x$ direction no Hall current occurs and for a magnetization pointing in the $y$ direction with $\Delta_{y}=0.0075 t$ we observe a maximum of the Hall conductance which occurs at the same energy as for the previous case of strictly out-of-plane magnetization $\Delta_{z}=0.0075 t$ but is smaller in magnitude. For a magnetization pointing in the $(1,1,0)$ direction with $\Delta_{x}=\Delta_{y}=0.0053 t$ (fulfilling $|\vec{\Delta}|=0.0075 t$ ) we find a conductance peak at the same position in energy but of different shape. If the magnitude of

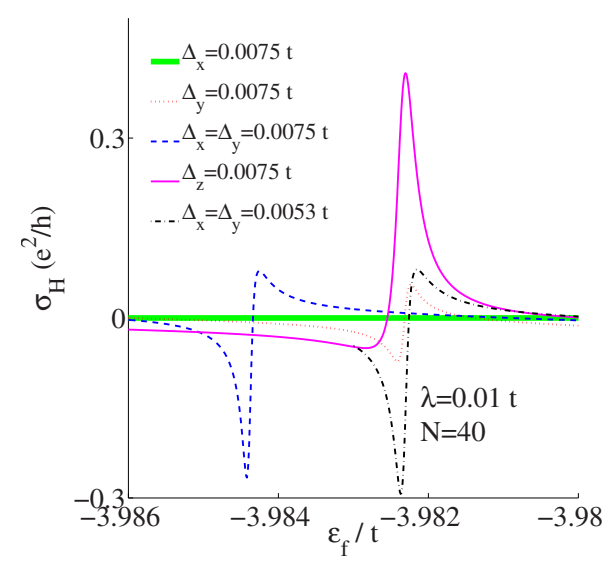

FIG. 8. (Color online) Hall conductance for a Rashba parameter of $\lambda=0.01 t$ and various directions of magnetization with the nonzero components of $\vec{\Delta}$ stated in the legend. For magnetization along the $x$ direction no Hall current occurs.

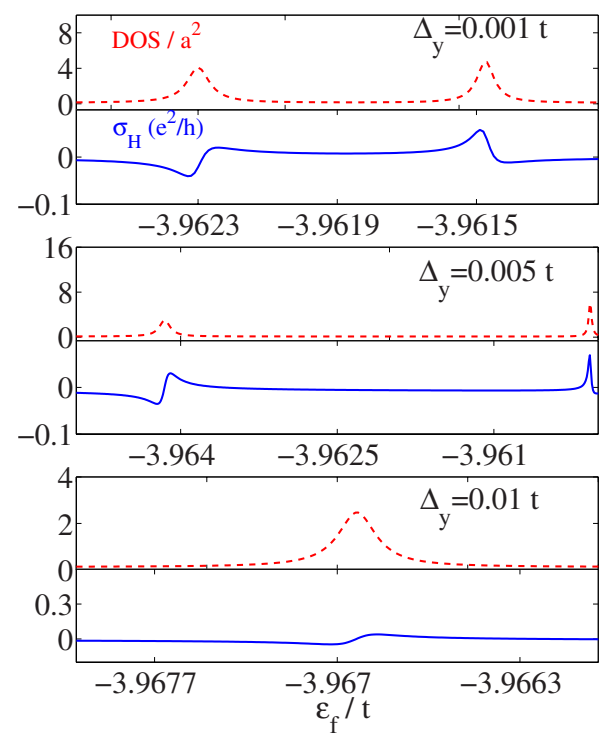

FIG. 9. (Color online) DOS (red dashed line) in units $1 / \mathrm{ta}^{2}$ and Hall conductance (blue solid line) in units of $e^{2} / h$ near the lower band edge for an in-plane magnetization polarized along the $y$ direction, for a system of linear size $N=30$ and for three different magnitudes of the Zeeman splitting. This picture is analogous to Fig. 4 for the case of a perpendicular magnetization. Even in the case of an in-plane magnetization we observe the correspondence between the extrema DOS and Hall conductance.

the Zeeman splitting is increased to $\Delta_{x}=\Delta_{y}=0.0075 t$ the peak approximately maintains its shape but is shifted toward lower energies. We focus now on an in-plane magnetization totally polarized along the $y$ direction. Figure 9 in the "inplane analog" of Fig. 4 and shows the correspondence between the extrema of the DOS and those of the Hall conductance for an in-plane magnetization totally magnetized along the $y$ direction for a system of linear size $N=30$ and for three different values of the magnitude of the Zeeman splitting. As in the case of a perpendicular magnetization, see Fig. 4, we observe a correspondence between the extrema of the DOS and the Hall conductance at the same Fermi energy.

In Fig. 10 we show the Hall conductance for an in-plane magnetization along the $y$ direction varying between $\Delta_{y}$ $=0.001 t$ and $\Delta_{y}=0.01 t$, and linear system sizes between $N$ $=30$ and 50. The Rashba parameter is again $\lambda=0.01 t$. Obviously, the position of Hall peaks shifts to lower energy with increasing systems size, analogously as in Fig. 5 for the case of a perpendicular magnetization. Moreover, for the smallest Zeeman coupling considered here, $\Delta_{y}=0.001 t$, the height of the peaks clearly grows with systems size while for the largest Zeeman coupling of $\Delta_{y}=0.01 t$ the opposite behavior is observed. However, we cannot outline any trend for intermediate values of the Zeeman coupling, see Figs. 10(b)-10(e). Another interesting finding is that the energetic position of the Hall signal coincides for in-plane and perpendicular magnetization of the same magnitude. This result is shown in Fig. 11, where the linear size of the system is $N=40$ and the Zeeman coupling is chosen to be $\Delta_{z}=\Delta_{y}=0.01 \mathrm{t}$. Here, in the two cases of perpendicular and in-plane magnetization the shape of the DOS and Hall conductance peaks varies but their maxima exactly coincide. The dependence of the Hall 


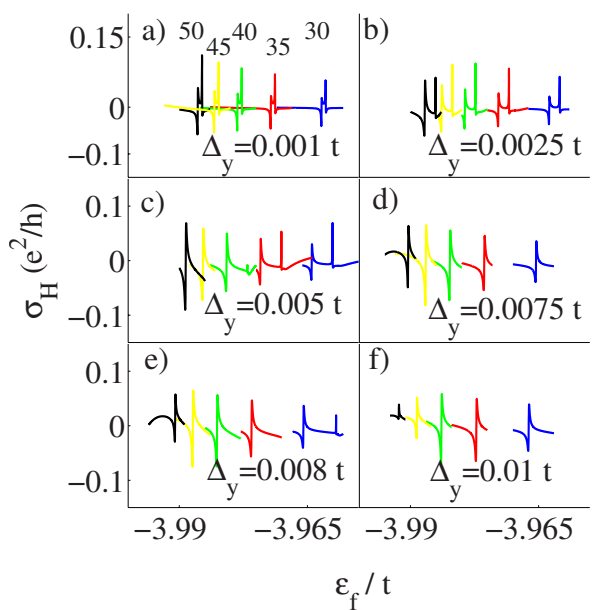

FIG. 10. (Color online) Hall conductance near the bottom of the band for a Rashba parameter of $\lambda=0.01 t$ in the case of an in-plane magnetization along the $y$ direction and for various Zeeman couplings $\Delta_{y}$. The linear system size varies from $N=30$ (blue, right), $N=35$ (red), $N=40$ (green), $N=45$ (yellow), and $N=50$ (black, left). This picture can be directly compared with Fig. 5 for the case of a perpendicular magnetization.

conductance on direction and magnitude of the in-plane magnetization is to be explored further in the future.

\section{B. Magnetically disordered systems}

So far we have studied homogeneously magnetized systems with each lattice site carrying a magnetic ion whose spin provides a Zeeman field for the carrier spins. We now consider the case where only a given fraction $\nu$ of lattice sites is occupied by a magnetic ion. This scenario accounts for the situation in real ferromagnetic semiconductor nanostructures. We will concentrate again on magnetizations along the $z$ direction perpendicular to the plane of the 2DEG. To be specific, we choose at random a given fraction of lattice sites to be occupied with a magnetic ion and average
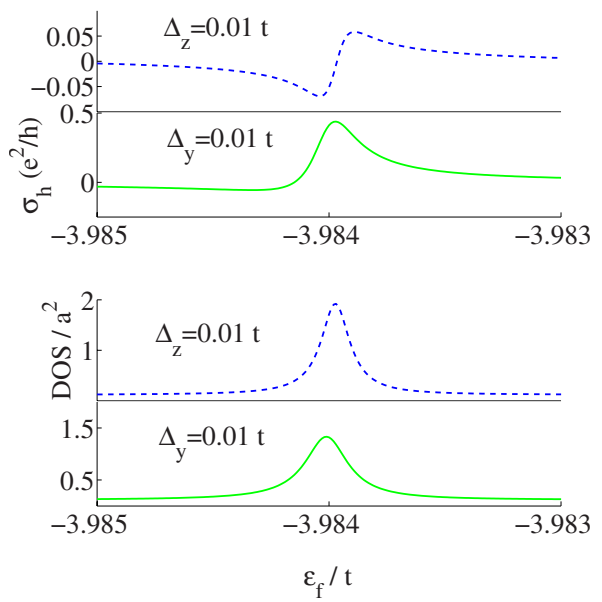

FIG. 11. (Color online) Hall conductance and DOS for a system of linear dimension $N=40$ and for a perpendicular magnetization of amplitude $\Delta_{z}=0.01 t$ (blue dashed line) and an in-plane magnetization $\Delta_{y}=0.01 t$ (green solid line).

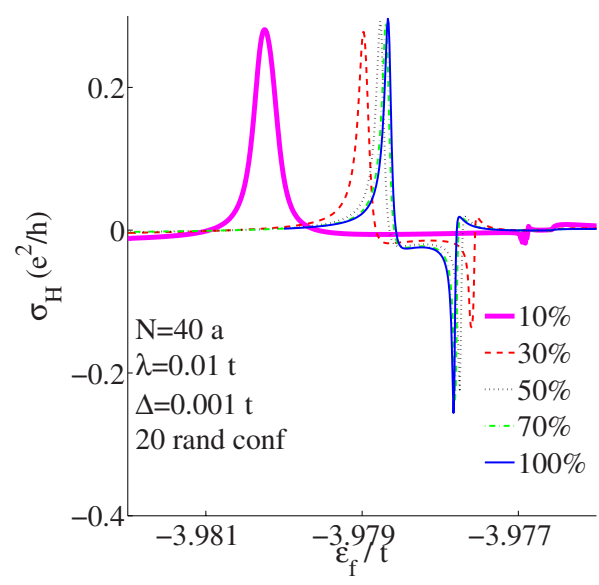

FIG. 12. (Color online) Hall conductance close to the lower band edge for different fractions of magnetically occupied sites for a Rashba parameter of $\lambda=0.01 t$ and an average magnetization of $\Delta=0.001 t$. The linear size of the system is $N=40$.

our results for the Hall conductivity over typically 20 of such disorder realizations, which, by inspection of the data, turns out to be sufficient. In order to facilitate the comparison with our previous results for magnetically homogeneous systems we adjust the magnitude of each local coupling $\Delta_{m, n}$ such that the average Zeeman coupling $\Delta:=\left(\Sigma_{m, n} \Delta_{m, n}\right) / N^{2}$ is constant, i.e., $\Delta_{m, n}=\Delta / \nu$. Figure 12 shows the Hall conductance for different fractions of magnetically occupied sites for a Rashba parameter of $\lambda=0.01 t$ and an average magnetization of $\Delta=0.001 t$. The data are averaged over 20 random disorder configurations. Figure 13 shows the dependence of the Hall conductance both on the fraction of magnetized sites as well as on system size. Again, the energetic position of the hall maxima moves toward lower energies with increasing system size, cf. Figs. 5 and 10. Moreover, for fractions $\nu$ $=0.3, \ldots, 0.9$ of magnetized sites, the position of the Hall peaks is very close to that of the uniform magnetization, $\nu=1$, and the height of peaks is approximately constant in all cases. This is different from the smallest fraction considered

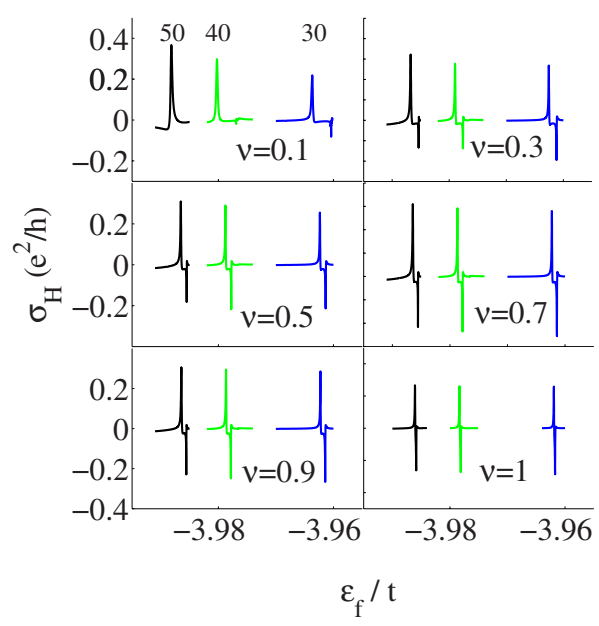

FIG. 13. (Color online) Hall conductance maxima for systems of linear dimension $N=30$ (blue, right), $N=40$ (green), $N=50$ (black, left) and for several fractions of magnetically occupied sites. 
here, $\nu=0.1$, where height increases with system size and also the energetic positions differ from the other cases.

\section{CONCLUSIONS AND OUTLOOK}

Anomalous Hall transport is still the subject of a longstanding theoretical discussion. In the present paper we have reported on the, to our knowledge, investigation of this phenomenon using the Landauer-Büttiker formalism. Specifically, we have studied a two-dimensional electron gas with Rashba spin-orbit coupling and a magnetization provided by localized magnetic moments. Our system is described by a discretized tight-binding model in a four-terminal geometry. We have considered both the case of ballistic transport in disorder-free systems with homogeneous magnetization as well as magnetically disordered systems. In the former case we have also distinguished between different directions of the magnetization. In particular, a magnetization lying entirely in the plane of the 2DEG can also be interpreted as a genuine magnetic field which couples, in a strictly twodimensional system, only to the spin of the electron but not to its orbital degrees of freedom.

In particular, we have demonstrated numerically a close connection between singularities in the density of states and peaks in the Hall conductance close to the lower band edge. Consistent with previous theoretical studies based on diagrammatic perturbation expansions, these peaks occur at Fermi energies where only the lower dispersion branch is occupied. ${ }^{18,20}$ Moreover, for large magnetization the Hall conductance is, along with the density of states, suppressed. This numerical finding can be understood from analytical properties of the underlying model in the limit of an infinite system.

Future investigation will include a more detailed understanding of anomalous Hall transport in the presence of an in-plane magnetization and the effects of magnetic disorder.

\section{ACKNOWLEDGMENT}

This work was supported by the SFB 689 "Spin Phenomena in Reduced Dimensions."

\section{APPENDIX: GREEN'S FUNCTION OF A SEMI-INFINITE LEAD}

Here we give some more technical details regarding the calculation of the Green's function of Eq. (21) for a semiinfinite lead. For a semi-infinite noninteracting lead with hard wall confinement and a constant width $L$, the transverse wave functions are

$$
\tilde{\chi}_{m}(y)=\sqrt{\frac{2}{L}} \sin \left(\frac{m \pi y}{L}\right)
$$

or

$$
\chi_{m}\left(y_{j}\right)=\sqrt{\frac{2}{M+1}} \sin \left(\frac{m \pi j}{M+1}\right),
$$

where $y_{i}=j a$ and $M$ is the number of sites in the transverse direction, such that $L=(M+1) a$. The longitudinal lattice wave functions are

$$
\phi_{k}(x)=\sqrt{\frac{2}{L}} \sin (k x),
$$

which by substituting $x=a$, which means that we are considering points $x$ at the first slice of the semi-infinite lead, transforms into

$$
\phi_{k}(x=a)=\sqrt{\frac{2 a}{L}} \sin (k a) .
$$

Finally, at the first slice $x=a$ the total wave function reads

$$
\psi_{m, k}=\sqrt{\frac{2 a}{L}} \chi_{m}(y) \sin (k a),
$$

which is an eigenfunction of the Hamiltonian $H_{0} \psi_{m, k}$ $=E_{m, k} \psi_{m, k}$, where the dispersion relation is given by

$$
E_{m, k}=2 t\left(1-\cos \frac{m \pi}{M+1}\right)+2 t[1-\cos (k a)] .
$$

Inserting the wave function in the eigenfunctions expression of the Green's function, see Ref. 25, the Green's function at the first slice $x=a$ is

$$
G^{r}\left(a, y_{i} ; a, y_{j}\right)=\frac{2 a}{L} \sum_{m, k>0} \frac{\chi_{m}\left(y_{i}\right) \chi_{m}^{*}\left(y_{j}\right) \sin ^{2}(k a)}{E-E_{m, k}+i \eta} .
$$

In the limit $L \rightarrow \infty$, we may replace the sum over $k$ by the integral $(L / \pi) \int_{0}^{\pi / a} d k$ and substitute $k a=\theta$ to obtain

$$
\begin{aligned}
G^{r}\left(a, y_{i} ; a, y_{j}\right)= & 1 / \pi t \sum_{m} \chi_{m}\left(y_{i}\right) \chi_{m}^{*}\left(y_{j}\right) \\
& \times \int_{0}^{\pi} d \theta \sin ^{2} \theta /(Q+\cos \theta+i \eta),
\end{aligned}
$$

where we made the following replacement

$$
Q=\frac{E}{2 t}-2+\cos \frac{m \pi}{M+1} .
$$

In Eq. (A8) the integrand function $\sin ^{2} \theta /(Q+\cos \theta+i \eta)$ is an even function of $\theta$, therefore the integral can be written as a symmetric integral from $-\pi$ to $\pi$. By writing in the integral the sine and cosine functions in terms of their exponential form and performing the substitution $z=\exp (i \theta)$, the integral turns into a closed contour integral along the unit circle in the complex plane, allowing us to write the Green's function as 


$$
\begin{aligned}
G^{r}\left(a, y_{i} ; a, y_{j}\right)= & 1 /(2 \pi i t) \sum_{m} \chi_{m}\left(y_{i}\right) \chi_{m}^{*}\left(y_{j}\right) \\
& \times \int_{C} d z\left(1-z^{2}\right) /\left(z^{2}+2 z Q+1\right),
\end{aligned}
$$

(A10)

where $\int_{C}$ stands for the integration on a closed circuit of radius $|z|=1$. By solving the integral with the use of the theorem of residues, we obtain

$$
\begin{aligned}
G^{r}\left(a, y_{i} ; a, y_{j}\right)= & 1 /(2 \pi i t) \sum_{m} \chi_{m}\left(y_{i}\right) \chi_{m}^{*}\left(y_{j}\right) 2 \pi i R_{z_{0}} \\
& \times\left(1-z^{2}\right) /\left(z^{2}+2 z Q+1\right),
\end{aligned}
$$

where $R_{z_{0}}$ indicates the residual calculated at the pole $z=z_{0}$ which depends on $Q$, i.e.,

$$
z_{0}=\left\{\begin{array}{ll}
-Q+\sqrt{Q^{2}-1} & \text { for } Q>1 \\
-Q-\sqrt{Q^{2}-1} & \text { for } Q<-1 \\
-Q+i \sqrt{1-Q^{2}} & \text { for }|Q| \leq 1
\end{array} .\right.
$$

Inserting the pole in Eq. (A11), we obtain the final expression for the Green's function

$$
G^{r}\left(a, y_{i} ; a, y_{j}\right)=\frac{1}{t} \sum_{m} \chi_{m}\left(y_{i}\right) \chi_{m}^{*}\left(y_{j}\right) F(Q)
$$

with

$$
F(Q)=\left\{\begin{array}{ll}
Q-\sqrt{Q^{2}-1} & \text { for } Q>1 \\
Q+\sqrt{Q^{2}-1} & \text { for } Q<-1 \\
Q-i \sqrt{1-Q^{2}} & \text { for }|Q| \leq 1
\end{array} .\right.
$$

${ }^{1}$ R. Karplus and J. M. Luttinger, Phys. Rev. 95, 1154 (1954).

${ }^{2}$ J. Smit, Physica (Amsterdam) 21, 877 (1955).

${ }^{3}$ L. Berger, Phys. Rev. B 2, 4559 (1970).

${ }^{4}$ P. Nozieres and C. Lewiner, J. Phys. (Paris) 34, 901 (1973).

${ }^{5}$ T. Jungwirth, Q. Niu, and A. H. MacDonald, Phys. Rev. Lett. 88, 207208 (2002).

${ }^{6}$ J. Sinova, T. Jungwirth, and J. Cerne, Int. J. Mod. Phys. B 18, 1083 (2004).

${ }^{7}$ N. A. Sinitsyn, J. Phys.: Condens. Matter 20, 023201 (2008).

${ }^{8}$ A. H. MacDonald, P. Schiffer, and N. Samarth, Nature Mater. 4, 195 (2005).

${ }^{9}$ T. Jungwirth, J. Sinova, J. Masek, and A. H. MacDonald, Rev. Mod. Phys. 78, 809 (2006).

${ }^{10}$ E. I. Rashba, Fiz. Tverd. Tela (Leningrad) 2, 1224 (1960) [Sov. Phys. Solid State 2, 1109 (1960)]; Y. A. Bychkov and E. I. Rashba, J. Phys. C 17, 6039 (1984).

${ }^{11}$ D. Culcer, A. H. MacDonald, and Q. Niu, Phys. Rev. B 68, 045327 (2003).

${ }^{12}$ V. K. Dugaev, P. Bruno, M. Taillefumier, B. Canals, and C. Lacroix, Phys. Rev. B 71, 224423 (2005).

${ }^{13}$ N. A. Sinitsyn, Q. Niu, J. Sinova, and K. Nomura, Phys. Rev. B 72, 045346 (2005).

${ }^{14}$ S. Y. Liu and X. L. Lei, Phys. Rev. B 72, 195329 (2005).

${ }^{15}$ S. Y. Liu, N. J. M. Horing, and X. L. Lei, Phys. Rev. B 74, 165316 (2006).

${ }^{16}$ S. Onoda, N. Sugimoto, and N. Nagaosa, Phys. Rev. Lett. 97, 126602 (2006).

${ }^{17}$ J.-I. Inoue, T. Kato, Y. Ishikawa, H. Itoh, G. E. W. Bauer, and L. W. Molenkamp, Phys. Rev. Lett. 97, 046604 (2006).

${ }^{18}$ T. Kato, Y. Ishikawa, H. Itoh, and J.-I. Inoue, New J. Phys. 9, 350 (2007).

${ }^{19}$ M. F. Borunda, T. S. Nunner, T. Luck, N. A. Sinitsyn, C. Timm, J. Wunderlich, T. Jungwirth, A. H. MacDonald, and J. Sinova, Phys. Rev. Lett. 99, 066604 (2007).
${ }^{20}$ T. S. Nunner, N. A. Sinitsyn, M. F. Borunda, V. K. Dugaev, A. A. Kovalev, A. Abanov, C. Timm, T. Jungwirth, J.-I. Inoue, A. H. MacDonald, and J. Sinova, Phys. Rev. B 76, 235312 (2007).

${ }^{21}$ T. S. Nunner, G. Zarand, and F. von Oppen, Phys. Rev. Lett. 100, 236602 (2008).

${ }^{22}$ A. A. Kovalev, K. Vyborny, and J. Sinova, Phys. Rev. B 78, 041305(R) (2008).

${ }^{23}$ A. A. Kovalev, Y. Tserkovnyak, K. Vyborny, and J. Sinova, Phys. Rev. B 79, 195129 (2009).

${ }^{24}$ J. Cumings, L. S. Moore, H. T. Chou, K. C. Ku, G. Xiang, S. A. Crooker, N. Samarth, and D. Goldhaber-Gordon, Phys. Rev. Lett. 96, 196404 (2006).

${ }^{25}$ S. Datta, Electronic Transport in Mesoscopic Systems (Cambridge University Press, Cambridge, 1997).

${ }^{26}$ D. K. Ferry and S. M. Goodnick, Transport in Nanostructures (Cambridge University Press, Cambridge, 1997).

${ }^{27}$ E. M. Hankiewicz, L. W. Molenkamp, T. Jungwirth, and J. Sinova, Phys. Rev. B 70, 241301(R) (2004).

${ }^{28}$ L. Sheng, D. N. Sheng, and C. S. Ting, Phys. Rev. Lett. 94, 016602 (2005).

${ }^{29}$ J. Li, L. Hu, and S.-Q. Shen, Phys. Rev. B 71, 241305(R) (2005).

${ }^{30}$ B. K. Nikolic, L. P. Zarbo, and S. Souma, Phys. Rev. B 72, 075361 (2005).

${ }^{31}$ B. K. Nikolic, S. Souma, L. P. Zarbo, and J. Sinova, Phys. Rev. Lett. 95, 046601 (2005).

${ }^{32}$ E. M. Hankiewicz, J. Li, T. Jungwirth, Q. Niu, S.-Q. Shen, and J. Sinova, Phys. Rev. B 72, 155305 (2005).

${ }^{33}$ S. I. Erlingsson and D. Loss, Phys. Rev. B 72, 121310(R) (2005).

${ }^{34}$ C. P. Moca and D. C. Marinescu, Phys. Rev. B 72, 165335 (2005).

${ }^{35}$ B. K. Nikolic, L. P. Zarbo, and S. Souma, Phys. Rev. B 73, 075303 (2006). 